\title{
Comparison of prasugrel and clopidogrel in patients with acute coronary syndrome undergoing percutaneous coronary intervention
}

This article was published in the following Dove Press journal:

Vascular Health and Risk Management

19 October 2009

Number of times this article has been viewed

\author{
Nicholas B Norgard' \\ Mazen Abu-Fadel ${ }^{2}$ \\ 'University at Buffalo, School of \\ Pharmacy and Pharmaceutical \\ Sciences, Buffalo, NY, USA; ${ }^{2}$ University \\ of Oklahoma Health Sciences Center, \\ Cardiovascular Section, Oklahoma \\ City, OK, USA
}

\begin{abstract}
Antiplatelet agents are the cornerstone of treatment for patients with acute coronary syndrome (ACS) undergoing percutaneous coronary intervention (PCI). Clopidogrel, when added to aspirin, has demonstrated considerable success at reducing thrombotic complications of ACS and/or PCI compared to aspirin alone and is standard of care for the management of patients with ACS and in patients undergoing PCI. Prasugrel is a novel thienopyridine antiplatelet agent recently approved for the treatment of patients with ACS undergoing PCI. Prasugrel provides greater and more consistent platelet inhibition than clopidogrel due to earlier and more extensive formation of its active metabolite. The enhanced platelet inhibition with prasugrel led to a reduction in major adverse cardiovascular events in patients with moderate to high risk ACS scheduled for PCI in the phase 3 TRITON-TIMI 38 trial. This benefit was seen more in patients suffering a STEMI and those with diabetes. However, this reduction in events was met with a significant increase in the risk of bleeding which overcame prasugrel's benefit in certain groups. Future studies with prasugrel are needed to determine its optimal utilization to minimize bleeding risks and evaluate its outcomes in ACS and safety profile in special patient populations.
\end{abstract}

Keywords: clopidogrel, prasugrel, percutaneous coronary intervention, acute coronary syndrome

\section{Introduction to the use and development of antiplatelet agents}

There is an evolving emphasis on optimizing antiplatelet therapy in the treatment of vascular disease and in patients undergoing vascular procedures. Platelets play an important role in cardiovascular disease both in the pathogenesis of atherosclerosis and in the development of acute thrombotic events. ${ }^{1}$ Their importance in vascular disease is indirectly confirmed by the benefit of antiplatelet agents in these disorders. Antiplatelet agents are the cornerstone of treatment for patients with acute coronary syndrome (ACS) undergoing percutaneous coronary intervention (PCI). ${ }^{2}$

For many years, aspirin has been the mainstay of antiplatelet drug therapy in vascular disease. As an antiplatelet agent, aspirin has been shown to greatly reduce major vascular adverse events. ${ }^{3}$ Its benefit has been linked to its ability to permanently acetylate cyclooxygenase $1(\mathrm{COX}-1)$, preventing the conversion of arachidonic acid to thromboxane A2 by the platelet. Thromboxane A2 is a strong platelet agonist and inhibiting its production decreases overall platelet aggregation at the site of the vascular injury. However, blocking this pathway 
has a limited overall effect on the various pathways of platelet activation. While aspirin markedly reduces arachidonic acid-induced platelet aggregation, platelet reactivity mediated by $\mathrm{COX}-1$ independent pathways (ie, collagen and adenosine diphosphate) can remain high. ${ }^{4,5}$ The need for inhibition of other platelet activation pathways has led to the development of additional antiplatelet drugs.

Adenosine diphosphate (ADP) is recognized as one of the most important mediators of both physiologic hemostasis and thrombosis. ${ }^{6-10}$ Development and utilization of the thienopyridines, agents that block ADP receptors on the platelet membrane, has represented a major advancement for treatment of patients with ACS undergoing PCI. The thienopyridines were the first alternative to aspirin for platelet inhibition. Since they inhibit platelets by different mechanisms, aspirin and thienopyridines work synergistically and are best when used in combination. Clopidogrel, when added to aspirin, has demonstrated considerable success at reducing thrombotic complications of ACS and/or PCI compared to aspirin alone. ${ }^{11}$ Despite clopidogrel's ability to effectively prevent myocardial infarction (MI), stroke or death in patients with ACS and those undergoing PCI, it has limitations that compromise its clinical utility: delayed onset of action, modest platelet inhibition and variability in patients' responses. ${ }^{12}$ These issues might explain why some patients continue to suffer from major adverse cardiovascular events while receiving dual oral antiplatelet therapy. Both the success and inadequacy of clopidogrel has prompted the search for similar but superior antiplatelet agents with more rapid onset of action and less patient variability.

Prasugrel is a novel antiplatelet agent that recently received Food and Drug Administration (FDA) approval for the treatment of patients with ACS undergoing PCI. Prasugrel is a thienopyridine with a chemical structure (Figure 1) and mechanism of action similar to that of clopidogrel. This review focuses on the pharmacologic and therapeutic differences between prasugrel and clopidogrel.

\section{Review of pharmacology, mode of action, pharmacokinetics of prasugrel, comparison to clopidogrel}

Administered orally, the thienopyridines are normally rapidly and extensively absorbed. Clopidogrel absorption is limited by the drug efflux transporter, P-glycoprotein (encoded by the ABCB1 gene). Polymorphisms of the ABCB1 gene contribute<smiles>COC(=O)/C=C1/CN(C(C(=O)OC)c2ccccc2Cl)N(C(C(=O)OC)c2ccccc2Cl)CCC1S</smiles>

Clopidogrel inactive carboxylic acid metabolite

(SR26334)

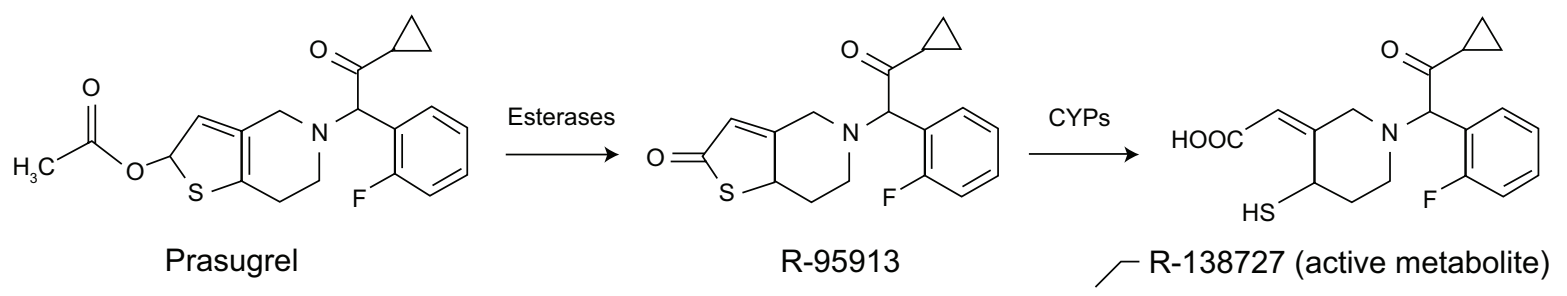

Figure I Chemical structure, and pathways leading to conversion to the active metabolites of clopidogrel and prasugrel. 
to the wide interpatient variability of clopidogrel bioavailability. Prasugrel's solubility decreases with increasing gastric $\mathrm{pH}$. Proton pump inhibitors (PPIs) increase gastric $\mathrm{pH}$, slowing the rate of dissolution and absorption of a prasugrel dose. ${ }^{13}$ However, this does not reduce the prasugrel's antiplatelet response. Clopidogrel absorption appears to be unaffected by PPIs, however its antiplatelet effect is reduced by the PPIs' effect on clopidogrel metabolism. H2 blockers do not affect either the absorption of either prasugrel or clopidogrel. ${ }^{14}$

The thienopyridines are prodrugs that must be metabolized in vivo into active form. Both prasugrel and clopidogrel require CYP450 metabolism for the generation of active metabolites, but the pathways leading to conversion to the active metabolites differ between the prodrugs (Figure 1). The majority of clopidogrel is metabolized into inactive metabolites by de-esterification. The remaining $15 \%$ of clopidogrel is converted to its active metabolite by $2 \mathrm{CYP}$ dependent steps (Figure 1). CYP1A2, CYP3A4/5, CYP2C9 and $\mathrm{CYP} 2 \mathrm{C} 19$ are considered to be the main contributors to active metabolite formation. ${ }^{15-18}$ Prasugrel is rapidly hydrolyzed by intestinal hydroxyesterases to an inactive thiolactone (R-95913), which is metabolized to the active metabolite, R-138727 by a single step, primarily by CYP3A4 and CYP2B6, and to a lesser extent by CYP2C9 and CYP2C19 (Figure 1). ${ }^{19} \mathrm{CYP} 3 \mathrm{~A}$ is considered the major enzyme responsible for active metabolite formation. Prasugrel metabolism is extensive as parent drug cannot be detected in plasma. The active metabolite is detected in human plasma within 15 minutes of administration and reaches maximum plasma concentration at approximately 30 minutes. ${ }^{20-22}$ The active metabolite R-138727 has an elimination half life of about 7.4 hours (range 2 to 15 hours). ${ }^{22}$ Unbound active metabolite is subsequently metabolized to inactive compounds by S-methylation (R-106583) and by cysteine conjugation (R-119251). ${ }^{22}$ The inactive metabolites are then primarily excreted in the urine. ${ }^{22}$

The need for bioactivation by CYPs puts the thienopyridines at risk for drug interactions with CYP inhibitors and inducers. Clopidogrel's active metabolite production and antiplatelet response are reduced by CYP3A4 and CYP2C19 inhibitors. ${ }^{21,23,24}$ Alternatively, St John's wort and rifampin, CYP3A4 inducers increase clopidogrel's antiplatelet response. ${ }^{24,25}$ In incubations of human liver microsomes, CYP3A4 inhibition with ketoconazole and CYP2B6 inhibition by monoclonal antibody significantly reduced prasugrel active metabolite formation. ${ }^{19}$ However, when tested in vivo, only the rate of active metabolism formation was reduced by ketoconazole, however there was no change in AUC. ${ }^{21}$ Thus, the interaction between prasugrel and CYP3A4 inhibitors appears not to be of clinical significance. In addition, no significant interaction was found between the CYP3A4 inducer rifampin and prasugrel. ${ }^{26}$ Inhibition of CYP2C19 and CYP2C9 have only minor effects on prasugrel metabolism. ${ }^{19}$

Genetic variation in the CYPs is an important determinant in clopidogrel active metabolite formation and antiplatelet response. Particularly, carriers of a reduced-function CYP2C19 allele have significantly lower levels of the active metabolite of clopidogrel, diminished platelet inhibition, and a higher rate of major adverse cardiovascular events. ${ }^{27,28}$ In healthy subjects and patients with stable atherosclerosis and ACS, genetic variation in CYP3A5, CYP2B6, CYP2C9, or CYP2C19 has no clinically significant effect on prasugrel as defined by active metabolite exposure levels. ${ }^{29-31}$

The active metabolites of prasugrel and clopidogrel produce their antiplatelet effect by irreversible inhibition of the platelet $\mathrm{P} 2 \mathrm{Y}_{12}$ receptor. The $\mathrm{P} 2 \mathrm{Y}_{12}$ receptor is responsible for the completion of ADP-induced platelet aggregation, augments platelet aggregation by other platelet agonists, such as collagen, thrombin, serotonin, epinephrine, and TxA2, and also amplifies the other functional consequences of activation, including granule release, $\mathrm{TxA}_{2}$ formation, and platelet proinflammatory and procoagulant activity. ${ }^{6-9}$ Several laboratory tests can be used to measure the thienopyridine effects on platelet activation and aggregation. In general, three methods are used to evaluate the pharmacodynamic response to the thienopyridines: light transmittance aggregometry (LTA), the VerifyNow P2Y ${ }_{12}$ assay, and the vasodilatorstimulated phosphoprotein (VASP) phosphorylation assay by flow cytometry. With LTA, ADP is used to stimulate platelet-rich plasma. Inhibition of platelet aggregation (IPA) is calculated as the percent decrease in aggregation during treatment when compared with baseline. ${ }^{32-35}$ There is no consensus definition for classifying antiplatelet response using this test. The VerifyNow $\mathrm{P}_{2} \mathrm{Y}_{12}$ assay is a point-of-care light transmittance assay which uses whole blood. ${ }^{36-38}$ This test is more specific than LTA for $\mathrm{P}_{2} \mathrm{Y}_{12}$ inhibition, as prostaglandin $\mathrm{E} 1$ is used to suppress the platelet $\mathrm{P} 2 \mathrm{Y}_{1}$ receptor response to ADP. The assay allows detection of absolute platelet reactive units (PRU) values and percent $\mathrm{P} 2 \mathrm{Y}_{12}$ receptor inhibition, which can be calculated as a percent change from baseline platelet aggregation. VASP phosphorylation is a test that directly measures the function of the $\mathrm{P} 2 \mathrm{Y}_{12}$ receptor. ${ }^{39}$ Dephosphorylation of VASP occurs following P2 $Y_{12}$ stimulation. Levels of VASP phosphorylation/ dephosphorylation thus reflect $\mathrm{P} 2 \mathrm{Y}_{12}$ inhibition/activation. 
VASP phosphorylation provides a selective index of platelet inhibition by the thienopyridines (platelet reactivity index [PRI]) and is not affected by other commonly used platelet inhibitors such as aspirin. Based on previous studies, patients are regarded as good responders to clopidogrel if $\mathrm{PRI}$ is $<50 \%$ and poor responders if $\mathrm{PRI}$ is $>50 \%$. Both in healthy subjects and coronary artery disease patients, there is a good agreement of antiplatelet response to thienopyridines measured by ADP-induced LTA, the VerifyNow P2 $\mathrm{Y}_{12}$ assay, and VASP phosphorylation. ${ }^{39-42}$

Several studies have demonstrated that a poor response to clopidogrel, defined as failure of the drug to achieve the expected suppression of platelet function as measured by any of the mentioned laboratory tests, identifies patients at risk of adverse clinical outcomes during treatment with clopidogrel..$^{32,43-45}$ The pharmacodynamic response to clopidogrel varies widely from subject to subject. Clopidogrel has been shown to produce $<5 \%$ platelet inhibition in some patients and up to $90 \%$ platelet inhibition in others with a roughly normal distribution. ${ }^{46}$ Approximately $30 \%$ of patients exhibit low platelet inhibition from clopidogrel are at risk for worsened cardiovascular outcomes. ${ }^{47,48}$ There are numerous mechanisms for a poor response to clopidogrel in patients, as genetic, metabolic, cellular, and clinical factors have been proposed. Higher doses of clopidogrel have been used to overcome hyporesponsiveness. ${ }^{49-56}$ While the mean platelet inhibition is greater with higher doses, significant interpatient variability remains and not all clopidogrel hyporesponsiveness can be overcome. ${ }^{49-56}$ Prasugrel was developed to produce a greater and more consistent platelet inhibition than clopidogrel.

Several studies have compared the platelet inhibitory capacity of prasugrel and clopidogrel. Preclinical studies indicate that prasugrel is approximately 10 -fold more potent than clopidogrel at inhibiting platelet aggregation, inhibiting thrombus formation, and prolonging bleeding times. ${ }^{57-59}$ In clinical studies, prasugrel has consistently demonstrated greater and more rapid platelet inhibition than clopidogrel in healthy subjects, patients with stable coronary artery disease (CAD) and acute coronary syndromes, and those undergoing PCI. In addition, subjects who are poor responders to clopidogrel respond adequately to prasugrel. ${ }^{20,60,61}$ Loading doses of prasugrel, $30 \mathrm{mg}$ to $75 \mathrm{mg}$, produce a dose-dependent platelet inhibition from $57 \%$ up to $90 \%$ inhibition of platelet aggregation. ${ }^{57,58}$ Prasugrel loading doses of 20, 30, 40, and $60 \mathrm{mg}$ have been shown to generate greater inhibition of platelet aggregation compared to a 300-mg clopidogrel loading dose. ${ }^{20,62-65}$ A 60 -mg prasugrel loading dose was shown to cause greater platelet inhibition when compared head-to-head with a $600-\mathrm{mg}$ clopidogrel loading dose. ${ }^{65,66}$ Along with a greater magnitude of platelet inhibition, prasugrel has a more rapid onset of platelet inhibition. Maximal effects of a 300-mg dose of clopidogrel are seen $>6$ hours after administration. A $600-\mathrm{mg}$ dose of clopidogrel achieves maximal inhibition 2 to 4 hours after administration. After a 60-mg dose of prasugrel, onset of platelet inhibition is seen after 15 minutes and reaches maximal effect after $\sim 1$ hour. Maintenance doses of 5, 10, and $15 \mathrm{mg}$ prasugrel also achieve consistent and significantly greater platelet inhibition than the standard 75-mg clopidogrel maintenance dose. .2,63,65,66 $^{-6}$ A 10-mg prasugrel maintenance dose was shown to cause greater platelet inhibition when compared head-to-head with a 150-mg clopidogrel maintenance dose. ${ }^{64}$ Changing from clopidogrel therapy to prasugrel maintenance therapy results in further reductions in maximal ADP-induced platelet aggregation early after switching. ${ }^{67}$

The antiplatelet effects of the active metabolites of prasugrel and clopidogrel are about equally potent in vitro. ${ }^{68}$ Subjects who are poor responders to clopidogrel have lower exposure to the active metabolite of clopidogrel than subjects who are normal responders. ${ }^{69}$ This implies that prasugrel's greater pharmacodynamic effect is related to the earlier and more extensive formation of the active metabolite of prasugrel compared with the equipotent active metabolite of clopidogrel.

\section{Efficacy and comparative studies with clopidogrel}

Prasugrel has been tested for efficacy in multiple studies. Three major clinical trials led to the approval of its use clinically. The Joint Utilization of Medications to Block Platelets Optimally (JUMBO-TIMI 26) was a phase 2, dose ranging, and safety trial of prasugrel vs clopidogrel in 904 patients undergoing elective or urgent PCI. After diagnostic angiography, patients were randomized to either clopidogrel (300 $\mathrm{mg}$ loading dose [LD] followed by $75 \mathrm{mg}$ daily) or to 1 of 3 doses of prasugrel, a lowdose regimen $40 \mathrm{mg} L \mathrm{LD}$ plus $7.5 \mathrm{mg}$ daily, an intermediate-dose regimen $60 \mathrm{mg} \mathrm{LD}$ plus $10 \mathrm{mg}$ daily, or a high-dose regimen 60 $\mathrm{mg}$ LD plus $15 \mathrm{mg}$ daily. Patients were treated with the study drug maintenance dose for 1 month with the co-administration of aspirin $325 \mathrm{mg}$ daily. The study found no significant difference in non-CABG bleeding between those receiving prasugrel and clopidogrel at 30 days (prasugrel $1.7 \%$ vs clopidogrel $1.2 \%$ ) or in TIMI major and minor bleeding. However, more TIMI minor bleeding events were detected in the prasugrel high-dose regimen group (3.6\%) compared to the intermediate-(1.5\%) or 
low-dose regimen $(2.0 \%)$ groups and to the clopidogrel group $(2.4 \%)$. The trial also showed a numerically lower incidence of major adverse cardiac events at 30 days in the prasugrel-treated patients, a difference that did not achieve statistical significance as a secondary efficacy end point. ${ }^{70}$

An additional evaluation of prasugrel vs clopidogrel was done in the The Prasugrel in Comparison to Clopidogrel for Inhibition of Platelet Activation and Aggregation (PRINCIPLE-TIMI 44) study in which 201 patients were randomized in a double-blind, two-phase crossover of prasugrel vs high-dose clopidogrel for planned PCI. During the first phase, prasugrel $60 \mathrm{mg}$ loading dose was compared to clopidogrel 600 mg loading dose with a primary end point of platelet inhibition at 6 hours. In the second phase, patients received $10 \mathrm{mg}$ maintenance dose of prasugrel for 14 days vs $150 \mathrm{mg}$ clopidogrel after the initial loading dose. On day 15 the patients were crossed over to the alternate maintenance treatment for an additional 2 weeks. As for the results, the IPA at 6 hours was significantly higher in the prasugrel group $(74.8 \% \pm 13 \%$ vs $31.8 \% \pm$ $21.1 \%$ ). The enhanced inhibitory effect of prasugrel was seen as early as 30 minutes after the loading dose. Patients treated with prasugrel also had more consistent levels of inhibition with significantly lower interpatient variability. In addition, the IPA after a 2 week maintenance dose was significantly greater in the prasugrel $(61.3 \% \pm 17.8 \%$ vs $46.1 \% \pm 21.3 \%)$. Of importance, prasugrel was well tolerated and only 2 patients in this group (none in the clopidogrel group) had TIMI minor bleeding before the cross over. The data from the PRINCIPLETIMI 44 confirmed that prasugrel is a more potent inhibitor of platelet aggregation in the setting of scheduled PCI. It also showed its ability to yield a rapid, high and consistent level of platelet inhibition. However, the question remained: Does a more potent platelet inhibition translate into a reduction in the atherothrombotic events clinically?

The Trial to Assess Improvement in Therapeutic Outcomes by Optimizing Platelet Inhibition with Prasugrel (TRITON)-TIMI 38 was a phase 3, randomized, double-blind, parallel group, multinational, clinical study designed to address this question and to evaluate for the first time in a large-scale clinical study whether a thienopyridine that results in a higher IPA results in improved clinical outcomes and is safe to use compared to the standard use of clopidogrel. ${ }^{71}$ The trial randomly assigned 13,608 patients with moderate to high risk ACS including 10,074 patients with unstable angina (UA) or non-ST segment elevation myocardial infarction (NSTEMI) and 3,534 patients with STEMI scheduled for PCI on a background of ASA therapy to clopidogrel $300 \mathrm{mg}$ loading and
$75 \mathrm{mg}$ maintenance vs prasugrel $60 \mathrm{mg}$ loading and $10 \mathrm{mg}$ maintenance. The loading dose was given any time between randomization and 1 hour after leaving the catheterization lab. Of importance is that the coronary anatomy had to be known to be suitable for PCI prior to randomization in all cases. Study subjects were followed at hospital discharge, 30 days, 90 days and at 3-month intervals for a total of 6 to 15 months. Key exclusion criteria included increased risk of bleeding, anemia, thrombocytopenia, known intracranial abnormalities, or the use of thienopyridines in the last 5 days. PCI at the time of randomization was performed on $99 \%$ of patients and $94 \%$ received at least one intracoronary stent. Drug eluting stents were used in $47 \%$ of subjects.

The primary efficacy end point for the study was a composite of the rate of cardiovascular death, nonfatal MI or non-fatal stroke during the follow-up period. This occurred in 781 patients $(12.1 \%)$ receiving clopidogrel compared to 643 patients $(9.9 \%)$ receiving prasugrel (hazard ratio [HR] for prasugrel vs clopidogrel $0.81 ; 95 \%$ CI 0.73 to 0.90 ; $P<0.001)$. This translates to a number needed to treat $(\mathrm{NNT})$ of forty-five. A significant benefit from prasugrel was seen by the first prespecified end point at 3 days (HR 0.82; CI, 0.71 to $0.96 ; P=0.01) .{ }^{72}$ In addition this benefit persisted through out the follow up period, which suggest a continued benefit of greater IPA during maintenance therapy after steady state IPA was achieved. Benefit was seen across the ACS spectrum. When patients were stratified by the presenting diagnosis, patients with UA and NSTEMI showed significant benefit with prasugrel compared to clopidogrel (HR 0.82; 95\% CI 0.73 to $0.93 ; P=0.002)$. An analysis of patients with STEMI $(\mathrm{n}=3534)$ also showed a significant reduction in events with prasugrel at 30 days (HR $0.68 ; 95 \%$ CI 0.54 to 0.87 ; $P=0.0017$ ) and at 15 months (HR $0.79 ; 95 \%$ CI 0.65 to 0.97 ; $P=0.02) .{ }^{73}$ There is some concern regarding the superiority of prasugrel due to the substantial inclusion of extra adjudicated MIs on top of investigator-reported MIs which resulted in an increase in the total reported primary outcomes. ${ }^{74}$ The clinical relevance of these 'extra MIs' is questionable. Additionally, the inclusion of these extra adjudicated events doubled the benefit of prasugrel over clopidogrel and are needed for maintain statistical significance.

The benefit of prasugrel, with regard to the primary end point, was seen both with and without the use of glycoprotein $\mathrm{IIb} / \mathrm{III}$ antagonists during the index hospitalization. ${ }^{75}$ The benefit with prasugrel vs clopidogrel tended to be greater among the 3,146 patients with diabetes (HR 0.70; 95\% CI, 0.58 to $0.85 ; P<0.001$ ) as compared to non diabetics (HR $0.86 ; 95 \% \mathrm{CI}, 0.76-0.98 ; P=0.02) .{ }^{76}$ The prasugrel group 
also showed a significant reduction in the secondary end point of death from cardiovascular causes, nonfatal MI, or urgent target vessel revascularization at 30 days (HR 0.78; 95\% CI 0.69 to $0.89 ; P<0.001$ ) and 90 days (HR 0.79; $95 \%$ CI 0.70 to $0.90 ; P<0.001$ ), as well as in the end point of death from cardiovascular causes, nonfatal MI, nonfatal stroke, or rehospitalization for ischemia (HR 0.84; 95\% CI 0.76 to $0.92 ; P<0.001)$. Significant reductions were seen in the prasugrel group compared with the clopidogrel group in the rates of $\mathrm{MI}(9.5 \%$ vs $7.3 \%$; HR 0.76 ; $95 \%$ CI 0.67 to $0.85 ; P<0.001$ ), urgent target-vessel revascularization (3.7\% vs $2.5 \%$; HR 0.66 ; $95 \%$ CI 0.54 to $0.81 ; P<0.001$ ), and stent thrombosis $(2.4 \%$ vs $1.1 \%$; HR $0.48 ; 95 \%$ CI 0.36 to $0.64 ; P<0.001)$. The findings on stent thrombosis were statistically significant irrespective of stent type. ${ }^{77}$

In a follow up study to TRITON-TIMI 38, Murphy et al hypothesized that prasugrel would reduce not only first events but also recurrent primary endpoint events and total events as compared to clopidogrel. ${ }^{78}$ This study is important since in the TRITON trial patients who experienced a component of the primary endpoint were censored from analysis following the initial event. Even though these patients continue to be followed during the trial, any additional events they have are generally not included in the primary endpoint efficacy analysis. This is important since in a real world setting these events are important for both the patient and the treating physician. In this analysis it was noted that patients with multiple events were older, had more co-morbidities at study entry including hypertension and diabetes and tended to be more females than males. Of importance, patients randomized to prasugrel were slightly older, less likely to be diabetic and more likely to have had multivessel PCI. Results of this analysis showed that among patients with an initial non-fatal event, second events were significantly reduced with prasugrel compared to clopidogrel (10.8 vs $15.4 \%$; HR $0.65 ; 95 \%$ CI 0.46 to $0.92 ; P=0.016)$, as was CV death following the non-fatal event (3.7 vs $7.1 \%$; HR 0.46 ; $95 \%$ CI 0.25 to $0.82 ; P=0.008)$. Overall, there was a reduction of 195 total primary efficacy events with prasugrel vs clopidogrel (rate ratio $0.79 ; 95 \% \mathrm{CI} 0.71$ to $0.87 ; P<0.001$ ). The reduction in second events with prasugrel was consistent in several key subgroups, including the elderly, gender, stent type, index event, and creatinine clearance. There was also a larger risk reduction in subsequent events in diabetics treated with prasugrel. These findings suggest that continued therapy with a regimen that provides higher levels of IPA remains important, even after an ischemic event has occurred. Indeed, intensive anti-platelet therapy seems to be of added benefit to those who have already had such an event. Based on the observation that total number of events in this analysis was higher with clopidogrel, it is possible that those patients with recurrent events may be more resistant to anti-platelet therapy, and/or more likely to be hyporesponders to platelet inhibition, which is associated with an increased risk of thrombotic events. ${ }^{77}$

\section{Safety and tolerability of prasugrel and clopidogrel}

The greater efficacy of prasugrel and its higher level of IPA come with a price. The most notable safety issue linked to the thienopyridines is their tendency to cause bleeding. Earlier studies showed signs of prasugrel's increased bleeding potential. $^{70}$ The large TRITON-TIMI 38 study provided more conclusive evidence that prasugrel is associated with excess bleeding relative to clopidogrel, irrespective of bleeding definition, seriousness, or location, and across most subgroups assessed. ${ }^{71}$ Among patients treated with prasugrel in the study, $146(2.4 \%)$ had TIMI major bleeding that was not related to coronary artery bypass grafting (CABG)as compared to 111 patients $(1.8 \%)$ treated with clopidogrel (HR 1.32; 95\% CI 1.03 to 1.68 ; number needed to harm $[\mathrm{NNH}]=167 ; P=0.03)$. This included a higher rate of life-threatening bleeding episodes. Additionally, in patients undergoing $\mathrm{CABG}$, there was a significantly higher rate of bleeding in the prasugrel group $(13.4 \%$ vs $3.2 \%$; NNH $=10)$. Thus, prasugrel should not be the drug of choice for patients in whom CABG surgery is anticipated or cannot be ruled out because the coronary anatomy is unknown. Fatal TIMI major bleeding occurred in a significantly higher number of patients treated with prasugrel $(0.4 \%$ vs $0.1 \% ; P=0.002)$. Intracranial bleeding was similar in both groups and occurred at a rate of $0.3 \%$ per group. It is interesting to note that approximately one-third of all bleeding events were recorded in the first day and almost half of all bleeding events were reported in the initial 10 days. Although bleeding can cause serious morbidity and mortality, the most critical consequences of bleeding (death, MI, and stroke), were included in the primary efficacy endpoint, where prasugrel was superior to clopidogrel. In addition, in a prespecified analysis of net clinical benefit, which included rates of efficacy endpoints and bleeding endpoints, prasugrel was noted to be superior to clopidogrel $(12.2 \%$ vs $13.9 \% ; P=0.004){ }^{73}$

The TRITON authors identified a few patient subgroups that did not have a net clinical outcome with prasugrel. ${ }^{71}$ These subgroups included patients with previous stroke or transient ischemic attacks (TIAs), patients 75 years of age or older, 
and patients weighing less than $60 \mathrm{~kg}$. These 3 subgroups had no clinical benefit from prasugrel mainly due to higher risk of bleeding with the drug. In particular, prasugrel's risk of bleeding in patients over 75 was similar to that in younger patients, however the outcomes secondary to bleeding in prasugrel-treated patients over 75 years of age were more serious in nature. Bleeding events in older patients were more often fatal or symptomatic intracranial hemorrhage. As prasugrel's efficacy is less clear in this subgroup of patients and appear to be at risk of more serious bleeding events, use of prasugrel should be discouraged in patients $\geq 75$ years of age. Patients without any of these risk factors had a greater efficacy with prasugrel without a significant difference in the rate of major bleeding and thus a substantially favorable net clinical benefit for the use of prasugrel. However, until additional work is done to better define the risks and benefits, the use of prasugrel should be avoided in patients with previous stroke/TIAs, age over 75 , or weight under $60 \mathrm{~kg}$.

As for the tolerability of prasugrel, more patients treated with prasugrel had to discontinue the drug due to adverse events related to bleeding compared to clopidogrel $(2.5 \%$ vs $1.4 \%)$. As for adverse events not related to bleeding in the TRITON study, $4.7 \%$ of patients treated with prasugrel and $5.0 \%$ of clopidogrel treated patients had to stop the drugs $(P=0.37)$ mainly due to severe thrombocytopenia $(0.3 \%$ in both groups), neutropenia which was significantly higher in the clopidogrel group $(<0.1 \%$ vs $0.2 \%)$, and colonic neoplasms due to increased diagnosis after GI bleeding in most of these patients which was significantly higher in the prasugrel group $(0.2 \%$ vs $0.1 \% ; P=0.03)(9)$.

\section{Conclusion}

Prasugrel represents an advance in oral antiplatelet therapy for patients with ACS treated with PCI. Clopidogrel, when added to aspirin, has been successful at reducing thrombotic complications of PCI and has been considered standard of care for several years. However, there are patients that remain at risk despite clopidogrel therapy, partly due to an inadequate pharmacodynamic response to the drug. Prasugrel, compared to clopidogrel, significantly decreases the risk for $\mathrm{MI}$ and stent thrombosis in patients with ACS undergoing PCI. This benefit was seen more in patients suffering a STEMI and those with diabetes. On the other hand, this reduction in events was met with a significant increase in the risk of bleeding. Prasugrel's benefit and risk are related to greater inhibition of platelet aggregation. Although excess fatal and non-fatal bleeding in prasugrel patients is obviously undesirable, it does not seem to outweigh prasugrel's benefit. The exception is in patients over the age of 75, weight under $60 \mathrm{~kg}$, and patients with a history of stroke or TIA. Prasugrel should be avoided in these patients. Future studies will determine whether lower prasugrel doses can be used to reduce bleeding risk while maintaining clinical efficacy.

Compared to clopidogrel, prasugrel's scope of use is relatively limited. It is indicated for use in patients undergoing PCI for ACS, but only for those in whom the coronary anatomy is known and CABG is unlikely. The use of prasugrel upstream in the emergency room is not recommended because of the high potential for CABG related bleeding. This should not influence outcome in patients who undergo PCI quickly after presentation. What about patients that are delayed in going for PCI? We know that upstream initiation of clopidogrel is advantageous as it shows benefit within 24 hours of initiation. ${ }^{79,80}$ It is still unknown whether withholding prasugrel treatment until PCI has negative consequences when the procedure is delayed. There is also no indication for its use in elective PCI and stable CAD. Future studies are still needed to define further the optimal use of prasugrel.

\section{Disclosures}

The authors declare no conflicts of interest.

\section{References}

1. Davi G, Patrono C. Platelet activation and atherothrombosis. $N$ Engl $J$ Med. 2007;357(24):2482-2494.

2. King SB 3rd, Smith SC Jr, Hirshfeld JW Jr, et al. 2007 Focused Update of the ACC/AHA/SCAI 2005 Guideline Update for Percutaneous Coronary Intervention: a report of the American College of Cardiology/American Heart Association Task Force on Practice Guidelines: 2007 Writing Group to Review New Evidence and Update the ACC/AHA/SCAI 2005 Guideline Update for Percutaneous Coronary Intervention, Writing on Behalf of the 2005 Writing Committee. Circulation. 2008;117(2):261-295.

3. Collaborative overview of randomised trials of antiplatelet therapy-I: Prevention of death, myocardial infarction, and stroke by prolonged antiplatelet therapy in various categories of patients. Antiplatelet Trialists' Collaboration. BMJ. 1994;308(6921):81-106.

4. Frelinger AL 3rd, Furman MI, Linden MD, et al. Residual arachidonic acid-induced platelet activation via an adenosine diphosphate-dependent but cyclooxygenase-1- and cyclooxygenase2-independent pathway: a 700-patient study of aspirin resistance. Circulation. 2006;113(25):2888-2896.

5. Ohmori T, Yatomi Y, Nonaka T, et al. Aspirin resistance detected with aggregometry cannot be explained by cyclooxygenase activity: involvement of other signaling pathway(s) in cardiovascular events of aspirin-treated patients. J Thromb Haemost. 2006;4(6):1271-1278.

6. Gachet C. Regulation of platelet functions by P2 receptors. Annu Rev Pharmacol Toxicol. 2006;46:277-300.

7. Gachet C. $\mathrm{P} 2$ receptors, platelet function and pharmacological implications. Thromb Haemost. 2008;99(3):466-472.

8. Gachet C. ADP receptors of platelets and their inhibition. Thromb Haemost. 2001;86(1):222-232.

9. Gachet C, Léon C, Hechler B. The platelet P2 receptors in arterial thrombosis. Blood Cells Mol Dis. 2006;36(2):223-227. 
10. Hechler B, Cattaneo M, Gachet C. The P2 receptors in platelet function. Semin Thromb Hemost. 2005;31(2):150-161.

11. Yusuf S, Zhao F, Mehta SR, Chrolavicius S, Tognoni G, Fox KK. Effects of clopidogrel in addition to aspirin in patients with acute coronary syndromes without ST-segment elevation. $N$ Engl J Med. 2001;345(7):494-502.

12. Norgard NB, Abu-Fadel M. Future prospects in anti-platelet therapy: a review of potential $\mathrm{P} 2 \mathrm{Y}_{12}$ and thrombin receptor antagonists. Recent Pat Cardiovasc Drug Discov. 2008;3(3):194-200.

13. Small DS, Farid NA, Payne CD, et al. Effects of the proton pump inhibitor lansoprazole on the pharmacokinetics and pharmacodynamics of prasugrel and clopidogrel. J Clin Pharmacol. 2008;48(4): 475-484.

14. Small DS, Farid NA, Li YG, et al. Effect of ranitidine on the pharmacokinetics and pharmacodynamics of prasugrel and clopidogrel. Curr Med Res Opin. 2008;24(8):2251-2257.

15. Savi P, Pereillo JM, Uzabiaga MF, et al. Identification and biological activity of the active metabolite of clopidogrel. Thromb Haemost. 2000;84(5):891-896.

16. Savi P, Combalbert J, Gaich C, et al. The antiaggregating activity of clopidogrel is due to a metabolic activation by the hepatic cytochrome P450-1 A. Thromb Haemost. 1994;72(2):313-317.

17. Lins R, Broekhuysen J, Necciari J, Deroubaix X. Pharmacokinetic profile of 14C-labeled clopidogrel. Semin Thromb Hemost. 1999;25 Suppl 2:29-33.

18. Kurihara A HK, Kazui M, Ishizuka T, Farid NA, Ikeda T. In vitro metabolism of antiplatelet agent clopidogrel: cytochrome $\mathrm{P} 450$ isoforms responsible for two oxidation steps involved in active metabolite formation. Drug Metab Rev. 2005;37(Suppl 2):99.

19. Rehmel JL, Eckstein JA, Farid NA, et al. Interactions of two major metabolites of prasugrel, a thienopyridine antiplatelet agent, with the cytochromes P450. Drug Metab Dispos. 2006;34(4):600-607.

20. Brandt JT, Payne CD, Wiviott SD, et al. A comparison of prasugrel and clopidogrel loading doses on platelet function: magnitude of platelet inhibition is related to active metabolite formation. Am Heart J. 2007;153(1):66.e9-e16.

21. Farid NA, Payne CD, Small DS, et al. Cytochrome P450 3 A inhibition by ketoconazole affects prasugrel and clopidogrel pharmacokinetics and pharmacodynamics differently. Clin Pharmacol Ther. 2007;81(5):735-741.

22. Farid NA, Smith RL, Gillespie TA, et al. The disposition of prasugrel, a novel thienopyridine, in humans. Drug Metab Dispos. 2007;35(7):1096-1104.

23. Gilard M, Arnaud B, Cornily JC, et al. Influence of omeprazole on the antiplatelet action of clopidogrel associated with aspirin: the randomized, double-blind OCLA (Omeprazole CLopidogrel Aspirin) study. J Am Coll Cardiol. 2008;51(3):256-260.

24. Lau WC, Gurbel PA, Watkins PB, et al. Contribution of hepatic cytochrome P450 3 A4 metabolic activity to the phenomenon of clopidogrel resistance. Circulation. 2004;109(2):166-171.

25. Lau W, Gurbel P, Carville D, et al. Saint Johns wort enhances clopidogrel responsiveness in clopidogrel resistant volunteers and patients by induction of CYP3A4 isoenzyme (abstr). $\mathrm{J} \mathrm{Am} \mathrm{Coll} \mathrm{Cardiol.}$ 2007;49(Suppl A):343A.

26. Farid NA, Jakubowski J, Payne CD, et al. Effect of rifampin on the pharmacokinetics and pharmacodynamics of prasugrel in healthy male subjects. Curr Med Res Opin. 2009;25(8):1821-1829.

27. Simon T, Verstuyft C, Mary-Krause M, et al. Genetic determinants of response to clopidogrel and cardiovascular events. $N$ Engl J Med. 2009;360(4):363-375.

28. Mega JL, Close SL, Wiviott SD, et al. Cytochrome p-450 polymorphisms and response to clopidogrel. $N$ Engl J Med. 2009;360(4):354-362.

29. Mega JL, Close SL, Wiviott SD, et al. Cytochrome P450 genetic polymorphisms and the response to prasugrel: relationship to pharmacokinetic, pharmacodynamic, and clinical outcomes. Circulation. 2009;119(19):2553-2560.
30. Varenhorst C, James S, Erlinge D, et al. Genetic variation of CYP2C19 affects both pharmacokinetic and pharmacodynamic responses to clopidogrel but not prasugrel in aspirin-treated patients with coronary artery disease. Eur Heart J. 2009;30(14):1744-1752.

31. Brandt JT, Close SL, Iturria SJ, et al. Common polymorphisms of CYP2C19 and CYP2C9 affect the pharmacokinetic and pharmacodynamic response to clopidogrel but not prasugrel. J Thromb Haemost. 2007;5(12):2429-2436.

32. Frere C, Cuisset T, Quilici J, et al. ADP-induced platelet aggregation and platelet reactivity index VASP are good predictive markers for clinical outcomes in non-ST elevation acute coronary syndrome. Thromb Haemost. 2007;98(4):838-843.

33. Geisler T, Langer $\mathrm{H}$, Wydymus $\mathrm{M}$, et al. Low response to clopidogrel is associated with cardiovascular outcome after coronary stent implantation. Eur Heart J. 2006;27(20):2420-2425.

34. Hochholzer W, Trenk D, Bestehorn HP, et al. Impact of the degree of peri-interventional platelet inhibition after loading with clopidogrel on early clinical outcome of elective coronary stent placement. $J$ Am Coll Cardiol. 2006;48(9):1742-1750.

35. Buonamici P, Marcucci R, Migliorini A, et al. Impact of platelet reactivity after clopidogrel administration on drug-eluting stent thrombosis. J Am Coll Cardiol. 2007;49(24):2312-2317.

36. Malinin A, Pokov A, Spergling M, et al. Monitoring platelet inhibition after clopidogrel with the VerifyNow-P2 $\mathrm{Y}_{12}(\mathrm{R})$ rapid analyzer: the VERIfy Thrombosis risk ASsessment (VERITAS) study. Thromb Res. 2007;119(3):277-284.

37. van Werkum JW, van der Stelt CA, Seesing TH, Hackeng CM, ten Berg JM. A head-to-head comparison between the VerifyNow P2 $\mathrm{Y}_{12}$ assay and light transmittance aggregometry for monitoring the individual platelet response to clopidogrel in patients undergoing elective percutaneous coronary intervention. J Thromb Haemost. 2006;4(11):2516-2518.

38. von Beckerath N, Pogatsa-Murray G, Wieczorek A, Sibbing D, Schomig A, Kastrati A. Correlation of a new point-of-care test with conventional optical aggregometry for the assessment of clopidogrel responsiveness. Thromb Haemost. 2006;95(5):910-911.

39. Aleil B, Ravanat C, Cazenave JP, Rochoux G, Heitz A, Gachet C. Flow cytometric analysis of intraplatelet VASP phosphorylation for the detection of clopidogrel resistance in patients with ischemic cardiovascular diseases. J Thromb Haemost. 2005;3(1):85-92.

40. Varenhorst C, James S, Erlinge D, et al. Assessment of P2Y(12) inhibition with the point-of-care device VerifyNow $\mathrm{P}_{2} \mathrm{Y}_{12}$ in patients treated with prasugrel or clopidogrel coadministered with aspirin. Am Heart J. 2009;157(3):562.e1-e9.

41. Jakubowski JA, Payne CD, Li YG, et al. The use of the VerifyNow $\mathrm{P} 2 \mathrm{Y}_{12}$ point-of-care device to monitor platelet function across a range of $\mathrm{P} 2 \mathrm{Y}_{12}$ inhibition levels following prasugrel and clopidogrel administration. Thromb Haemost. 2008;99(2):409-415.

42. Geiger J, Teichmann L, Grossmann R, et al. Monitoring of clopidogrel action: comparison of methods. Clin Chem. 2005;51(6):957-965.

43. Barragan P, Bouvier JL, Roquebert PO, et al. Resistance to thienopyridines: clinical detection of coronary stent thrombosis by monitoring of vasodilator-stimulated phosphoprotein phosphorylation. Catheter Cardiovasc Interv. 2003;59(3):295-302.

44. Bonello L, Paganelli F, Arpin-Bornet M, et al. Vasodilator-stimulated phosphoprotein phosphorylation analysis prior to percutaneous coronary intervention for exclusion of postprocedural major adverse cardiovascular events. J Thromb Haemost. 2007;5(8):1630-1636.

45. Blindt R, Stellbrink K, de Taeye A, et al. The significance of vasodilatorstimulated phosphoprotein for risk stratification of stent thrombosis. Thromb Haemost. 2007;98(6):1329-1334.

46. Gurbel PA, Bliden KP, Hiatt BL, O'Connor CM. Clopidogrel for coronary stenting: response variability, drug resistance, and the effect of pretreatment platelet reactivity. Circulation. 2003;107(23):2908-2913.

47. Matetzky S, Shenkman B, Guetta V, et al. Clopidogrel resistance is associated with increased risk of recurrent atherothrombotic events in patients with acute myocardial infarction. Circulation. 2004;109(25):3171-3175. 
48. Snoep JD, Hovens MM, Eikenboom JC, van der Bom JG, Jukema JW, Huisman MV. Clopidogrel nonresponsiveness in patients undergoing percutaneous coronary intervention with stenting: a systematic review and meta-analysis. Am Heart J. 2007;154(2):221-231.

49. Montalescot G, Sideris G, Meuleman C, et al. A randomized comparison of high clopidogrel loading doses in patients with non-ST-segment elevation acute coronary syndromes: the ALBION (Assessment of the Best Loading Dose of Clopidogrel to Blunt Platelet Activation, Inflammation and Ongoing Necrosis) trial. J Am Coll Cardiol. 2006;48(5):931-938.

50. von Beckerath N, Taubert D, Pogatsa-Murray G, Schömig E, Kastrati A, Schömig A. Absorption, metabolization, and antiplatelet effects of 300-, 600-, and 900-mg loading doses of clopidogrel: results of the ISAR-CHOICE (Intracoronary Stenting and Antithrombotic Regimen: Choose Between 3 High Oral Doses for Immediate Clopidogrel Effect) Trial. Circulation. 2005;112(19):2946-2950.

51. Lemesle G, Delhaye C, Sudre A, et al. Impact of high loading and maintenance dose of clopidogrel within the first 15 days after percutaneous coronary intervention on patient outcome. Am Heart J. 2009;157(2):375-382.

52. L'Allier PL, Ducrocq G, Pranno N, et al. Clopidogrel 600-mg double loading dose achieves stronger platelet inhibition than conventional regimens: results from the PREPAIR randomized study. J Am Coll Cardiol. 2008;51(11):1066-1172.

53. Cuisset T, Frere C, Quilici J, et al. Benefit of a 600-mg loading dose of clopidogrel on platelet reactivity and clinical outcomes in patients with non-ST-segment elevation acute coronary syndrome undergoing coronary stenting. J Am Coll Cardiol. 2006;48(7):1339-1345.

54. Angiolillo D, Fernández-Ortiz A, Bernardo E, et al. High clopidogrel loading dose during coronary stenting: effects on drug response and interindividual variability. Eur Heart J. 2004;25(21):1903-1910.

55. Angiolillo DJ, Bernardo E, Palazuelos J, et al. Functional impact of high clopidogrel maintenance dosing in patients undergoing elective percutaneous coronary interventions. Results of a randomized study. Thromb Haemost. 2008;99(1):161-168.

56. Angiolillo DJ, Costa MA, Shoemaker SB, et al. Functional effects of high clopidogrel maintenance dosing in patients with inadequate platelet inhibition on standard dose treatment. Am J Cardiol. 2008;101(4):440-445.

57. Asai F, Jakubowski JA, Naganuma H, et al. Platelet inhibitory activity and pharmacokinetics of prasugrel (CS-747) a novel thienopyridine P2Y12 inhibitor: a single ascending dose study in healthy humans. Platelets. 2006;17(4):209-217.

58. Jakubowski JA, Payne CD, Brandt JT, et al. The platelet inhibitory effects and pharmacokinetics of prasugrel after administration of loading and maintenance doses in healthy subjects. J Cardiovasc Pharmacol. 2006;47(3):377-384.

59. Matsushima N, Jakubowski JA, Asai F, et al. Platelet inhibitory activity and pharmacokinetics of prasugrel (CS-747) a novel thienopyridine P2Y12 inhibitor: a multiple-dose study in healthy humans. Platelets. 2006;17(4):218-226.

60. Weerakkody GJ, Jakubowski JA, Brandt JT, et al. Comparison of speed of onset of platelet inhibition after loading doses of clopidogrel versus prasugrel in healthy volunteers and correlation with responder status. Am J Cardiol. 2007;100(2):331-336.

61. Weerakkody GJ, Jakubowski JA, Brandt JT, Payne CD, Naganuma H, Winters KJ. Greater inhibition of platelet aggregation and reduced response variability with prasugrel versus clopidogrel: an integrated analysis. J Cardiovasr Pharmacol Ther. 2007;12(3):205-212.

62. Jakubowski JA, Winters KJ, Naganuma H, Wallentin L. Prasugrel: a novel thienopyridine antiplatelet agent. A review of preclinical and clinical studies and the mechanistic basis for its distinct antiplatelet profile. Cardiovasc Drug Rev. 2007;25(4):357-374.

63. Jernberg T, Payne CD, Winters KJ, et al. Prasugrel achieves greater inhibition of platelet aggregation and a lower rate of non-responders compared with clopidogrel in aspirin-treated patients with stable coronary artery disease. Eur Heart J. 2006;27(10):1166-1173.
64. Wiviott SD, Trenk D, Frelinger AL, et al. Prasugrel compared with high loading- and maintenance-dose clopidogrel in patients with planned percutaneous coronary intervention: the Prasugrel in Comparison to Clopidogrel for Inhibition of Platelet Activation and Aggregation-Thrombolysis in Myocardial Infarction 44 trial. Circulation. 2007;116(25):2923-2932.

65. Payne CD, Li YG, Small DS, et al. Increased active metabolite formation explains the greater platelet inhibition with prasugrel compared to high-dose clopidogrel. J Cardiovasc Pharmacol. 2007;50(5):555-562.

66. Wallentin L, Varenhorst C, James S, et al. Prasugrel achieves greater and faster $\mathrm{P} 2 \mathrm{Y}_{12}$ receptor-mediated platelet inhibition than clopidogrel due to more efficient generation of its active metabolite in aspirin-treated patients with coronary artery disease. Eur Heart J. 2008;29(1):21-30.

67. Payne CD, Li YG, Brandt JT, et al. Switching directly to prasugrel from clopidogrel results in greater inhibition of platelet aggregation in aspirin-treated subjects. Platelets. 2008;19(4):275-281.

68. Sugidachi A, Ogawa T, Kurihara A, et al. The greater in vivo antiplatelet effects of prasugrel as compared to clopidogrel reflect more efficient generation of its active metabolite with similar antiplatelet activity to that of clopidogrel's active metabolite. J Thromb Haemost. 2007;5(7):1545-1551.

69. Erlinge D, Varenhorst C, Braun OO, et al. Patients with poor responsiveness to thienopyridine treatment or with diabetes have lower levels of circulating active metabolite, but their platelets respond normally to active metabolite added ex vivo. J Am Coll Cardiol. 2008;52(24):1968-1977.

70. Wiviott SD, Antman EM, Winters KJ, et al. Randomized comparison of prasugrel (CS-747, LY640315), a novel thienopyridine $\mathrm{P}_{2} \mathrm{Y}_{12}$ antagonist, with clopidogrel in percutaneous coronary intervention: results of the Joint Utilization of Medications to Block Platelets Optimally (JUMBO)TIMI 26 trial. Circulation. 2005;111(25):3366-3373.

71. Wiviott SD, Braunwald E, McCabe $\mathrm{CH}$, et al. Prasugrel versus clopidogrel in patients with acute coronary syndromes. $N$ Engl J Med. 2007;357(20):2001-2015.

72. Antman EM, Wiviott SD, Murphy SA, et al. Early and late benefits of prasugrel in patients with acute coronary syndromes undergoing percutaneous coronary intervention: a TRITON-TIMI 38 (TRial to Assess Improvement in Therapeutic Outcomes by Optimizing Platelet InhibitioN with Prasugrel-Thrombolysis In Myocardial Infarction) analysis. J Am Coll Cardiol. 2008;51(21):2028-2033.

73. Montalescot G, Wiviott SD, Braunwald E, et al. Prasugrel compared with clopidogrel in patients undergoing percutaneous coronary intervention for ST-elevation myocardial infarction (TRITONTIMI 38): double-blind, randomised controlled trial. Lancet. 2009;373(9665):723-731.

74. Serebruany VL. The FDA Prasugrel Review: Adjudication of Myocardial Infarction Controversy. Cardiology. 2009;114(2):126-129.

75. O'Donoghue M, Antman EM, Braunwald E, et al. The efficacy and safety of prasugrel with and without a glycoprotein IIb/IIIa inhibitor in patients with acute coronary syndromes undergoing percutaneous intervention: a TRITON-TIMI 38 (Trial to Assess Improvement in Therapeutic Outcomes by Optimizing Platelet Inhibition With Prasugrel-Thrombolysis In Myocardial Infarction 38) Analysis. J Am Coll Cardiol. 2009;54(8):678-685.

76. Wiviott SD, Braunwald E, Angiolillo DJ, et al. Greater clinical benefit of more intensive oral antiplatelet therapy with prasugrel in patients with diabetes mellitus in the trial to assess improvement in therapeutic outcomes by optimizing platelet inhibition with prasugrel-Thrombolysis in Myocardial Infarction 38. Circulation. 2008;118(16):1626-1636.

77. Wiviott SD, Braunwald $\mathrm{E}, \mathrm{McCabe} \mathrm{CH}$, et al. Intensive oral antiplatelet therapy for reduction of ischaemic events including stent thrombosis in patients with acute coronary syndromes treated with percutaneous coronary intervention and stenting in the TRITON-TIMI 38 trial: a subanalysis of a randomised trial. Lancet. 2008;371(9621): 1353-1363. 
78. Murphy SA, Antman EM, Wiviott SD, et al. Reduction in recurrent cardiovascular events with prasugrel compared with clopidogrel in patients with acute coronary syndromes from the TRITON-TIMI 38 trial. Eur Heart J. 2008;29(20):2473-2479.

79. Mehta SR, Yusuf S, Peters RJ, et al. Effects of pretreatment with clopidogrel and aspirin followed by long-term therapy in patients undergoing percutaneous coronary intervention: the PCI-CURE study. Lancet. 2001;358(9281):527-533.
80. Yusuf S, Mehta S, Zhao F, et al. Early and late effects of clopidogrel in patients with acute coronary syndromes. Circulation. 2003;107(7):966-972.

\section{Publish your work in this journal}

Vascular Health and Risk Management is an international, peerreviewed journal of therapeutics and risk management, focusing on concise rapid reporting of clinical studies on the processes involved in the maintenance of vascular health; the monitoring, prevention and treatment of vascular disease and its sequelae; and the involvement of metabolic disorders, particularly diabetes. This journal is indexed on PubMed Central and MedLine. The manuscript management system is completely online and includes a very quick and fair peer-review system, which is all easy to use. Visit http://www.dovepress.com/ testimonials.php to read real quotes from published authors.

Submit your manuscript here: http://www.dovepress.com/vascular-health-and-risk-management-journal 